\title{
The Effects of WI-FI on College Students' Physical and Mental Health*
}

\author{
Yongmei Hou ${ }^{\mathrm{a}}$, Xuelin Li, Jinzhao Zhang, Wenling Xu, Yan Xie
}

Staff Room of Psychology, School of Humanity and Administration, Guangdong Medical University, Dongguan 523808, China

a352314640@qq.com

Keywords: WI-FI usage; physical and mental health; sub-health; college students

\begin{abstract}
Purpose: To understand college students' physical and mental health status and the use of WI-FI, and to explore the relationship between them. Method: A total of 1174 undergraduate students from 5 universities in Guangdong province were selected by stratified random method, and they were investigated by using personal general situation questionnaire (scl-90) and WI-FI questionnaire (WI-FI) compiled by themselves. Result: (1) The overall mental health of college students is good, but there are sub-health problems, mainly manifested as somatization, interpersonal sensitivity, compulsion, depression, anxiety, etc. (2) There are obvious somatic sub-health problems in college students, which are mainly manifested in pain and numbness of neck and lumbar spine, dry eyes, even pain and vision loss. (3) College students lack sufficient knowledge of WI-FI. 80.32\% of college students belong to high frequency WI-FI users, and 17.12\% are addicted to WI-FI. (4) Multivariate linear regression analysis results show that college students' mental health level (SCL - 90 total split) were the major influencing factors of the following aspects: the purpose of understanding of WI-FI, surfing the Internet, on the degree of psychological dependence on WI-FI, whether to install WI-FI software, mobile phone support WI-FI, degree of eyes, neck involvement, the degree of lumbar spine involvement, the places and time of WI-FI use, the WI-FI hours a day on average, and whether to use WI-FI access to the Internet in class time. Conclusion: College students have physical and mental sub-health problems, and the use of WI-FI has a significant impact on their physical and mental health.
\end{abstract}

\section{Introduction}

With the advantages of faster speed, better convenience, economy and safety than the cable network signal transmission, WI-FI (Wireless Fidelity) quickly got involved into the daily life of college students, leading a profound change in their way of life and providing great convenience for them to obtain knowledge, and also providing a broad space for their growth ${ }^{[1-3]}$. It can be said that today's college students live in a WI-FI environment.

College students are increasingly dependent on WI-FI. More than 80 percent of college students are reported to be high frequency WI-FI users. As a result, there is a significant reduction in the condition of psychological health of college students, which is mainly manifested in the cognitive narrow, distort, mood swings, easy to loneliness, depression, anxiety, sensitive, irritability, social disorder, uncontrollable behavior, and personality changes, etc ${ }^{[4-5]}$. At the same time, their health is declining year by year. In addition to the height and weight on the rise, standing long jump performance stable, the rest of the indicators (such as vital capacity, $50 \mathrm{~m}, 800 / 1000 \mathrm{~m}$, sit-ups, pull-ups in succession, crook proneness, etc.) are in decline year by year, while the incidence of overweight and obesity increased year by year.

Previous studies have shown that high frequency Internet use has some negative effects on college students' physical and mental health ${ }^{[7-8]}$. But most of these studies focused on the use of wired networks and related research on physical and mental health. Although that there is a connection, it has not been able to show is a direct correlation between covariant relationship, and it can't reflect what factors affect the physical and mental health in network use. As for the health effects of WI-FI, a special network environment, there are only a few descriptive studies.

Therefore, we urgently need to identify the factors influencing college students' physical and mental 
health in the use of WI-FI, so as to provide reference for the work of education.

\section{Research Objects and Methods}

\subsection{Research Objects}

Using stratified random sampling method to select 1300 undergraduates from five universities or colleges of Guangdong Institute of Finance, South China University of Technology Guangzhou College, Guangdong University of Technology, Guangdong Medical College, Dongguan Institute of Technology (the first three schools are a complete coverage of WI-FI, the later two are partly coverage of WI-FI) to give a questionnaire analysis. The research recycled 1174 effective questionnaires, with and effective rate of $90.3 \%$. Among them, 541 were boys and 633 were girls. There were 699 urban students, 216 students from the urban and rural integration department and 259 rural students; 240 science students, 392 engineering students, 511 arts students and 31 fine arts students; 162 outstanding students, 448 good students, 397 middle school students, 122 lower school students and 45 poor students; for their family conditions, there were 70 rich families, 530 well-off families, 516 ordinary families and 58 poor families.

\subsection{Research Tools}

\subsubsection{Symptom Checklist}

The research has a total of 90 projects, including somatization, coercion, interpersonal sensitivity, depression, anxiety, hostility, terror, paranoia, psychosis and other 10 factors. Using a likert scale of 1 to 5 , "1" stands for "there is no such thing" and "5" stands for "serious". The higher the score, the worse the symptoms and mental health. In this study, the Cronbach's a coefficient of each factor was $0.7736 \sim 0.8617$, and the Cronbach's a coefficient of the total table was 0.8904 . The retest reliability of each factor interval of one month is $0.7533 \sim 0.8419$, and the retest reliability of the total table interval of one month is 0.8847 .

\subsubsection{Self-compiled Personal General Information Questionnaire}

The content includes 8 items, including gender, age, grade, residence, school, major type, class rank and family economic status.

\subsubsection{Self-compiled Questionnaire on the Use of WI-FI in College Students}

19 items including the degree of understanding of WI-FI, WI-FI's most attractive aspects, personal phone support WI-FI access to the Internet, the main tool of Internet access of WI-FI, in class using WI-FI or not, the main place of Internet access with WI-FI, the main periods using WI-FI, average use WI-FI time every day, every day the number of connected WI-FI, main activities in using WI-FI, dormitory installed WI-FI signal transmitter or not, the PC installed free WI-FI Software, whether use WI-FI in public in a hurry, the affected degree on neck and shoulder, the degree of lumbar spine involvement, eyes involvement degree, the degree of psychological dependence on WI-FI, whether to use WI-FI access to the Internet as a way to avoid problems or ease the bad feelings, the causes of college students to be addicted to WI-FI.

\subsection{Data Processing}

SPSS20.0 was used to process the data, including descriptive statistics, single sample t test, independent sample t test, single factor variance analysis (ANOVA), multiple linear regression, etc.

\section{Results}

\subsection{The Use of WI-FI in College Students in This Group}

All students in this group use WI-FI every day. 92.84\% of the students have some knowledge of WI-FI, but $66.27 \%$ of them do not. $95.23 \%$ of students use WI-FI because of its convenience and economy. $95.06 \%$ of students use mobile phones that support WI-FI. 32.62\% of students never use WI-FI in class. $81.35 \%$ of students use smart phones as the main tool of WI-FI. $72.83 \%$ of students 
regard dormitory as the primary place for Internet access. $75.12 \%$ of students' dormitories are equipped with WI-FI signal transmitters. $55.28 \%$ of personal computers have free WI-FI software. $51.45 \%$ of students are also eager to use WI-FI in public places. $62.95 \%$ of students spend most of their time on the Internet at night. $80.32 \%$ of students spend more than 3 hours online on average every day. $67.29 \%$ of students connect to WI-FI more than 3 times a day. 86.88 percent of students spend most of their time online playing games, chatting and watching movies. $45.92 \%$ of students often increase their time and investment in using WI-FI in order to achieve a certain pleasure, and $37.99 \%$ of students often reduce their interest in other extracurricular activities due to the use of WI-FI. 40.80 percent of the students used WI-FI as a way to escape their worries and reduce their bad mood, while 17.12 percent of the students had a strong dependence on WI-FI.

\subsection{The General Physical Health of College Students in This Group}

$76.41 \%$ of the students had obvious fatigue in the neck, $66.02 \%$ had stiffness or even pain in the lumbar spine, $47.19 \%$ had dry eyes or even pain, and 55.71\% had obvious vision loss.

\subsection{Mental Health Status of College Students in This Group}

\subsubsection{Overall Situation}

In this group, $28.7 \%$ of college students had a total SCL-90 score $>2$ (positive symptoms); the percentage of SCL-90 factors with a score of $>2$ (positive symptoms) were: somatization (35.2\%), forced (42.6) \%), interpersonal sensitivity (43.3\%), depression (27.1\%), anxiety (24.2\%), hostility (15.3\%), terror (10.6\%), paranoia (8.7\%), and psychosis $(6.4 \%)$.

\subsubsection{The Mental Health Status of College Students in This Group Compared with That of College} Students Nationwide

Table 1 shows, this group of students in SCL - 90 of somatization and sensitive interpersonal relationship between the two factors of college students is significantly higher than the national norm, obsessive-compulsive disorder, depression, anxiety, hostility and terror five factors scored significantly lower than the national norm.

Table 1 The Mental Health Status of College Students in This Group Compared with That of College Students Nationwide $(\mathrm{M} \pm \mathrm{SD})$

\begin{tabular}{c|c|c|c|c}
\hline & Group(n=1167) & $\begin{array}{c}\text { Nationwide[10] } \\
(\mathrm{n}=9941)\end{array}$ & \multirow{2}{*}{$t$} & \\
& $\mathrm{M} \pm \mathrm{SD}$ & $\mathrm{M} \pm \mathrm{SD}$ & & .003 \\
\cline { 2 - 4 } & $1.49 \pm 0.47$ & $1.45 \pm 0.49$ & 2.946 & .000 \\
\hline Somatization & $1.69 \pm 0.55$ & $1.98 \pm 0.63$ & -17.907 & .000 \\
\hline OCD & $1.96 \pm 0.54$ & $1.88 \pm 0.63$ & 4.879 & .000 \\
\hline Interpersonal sensitivity & $1.50 \pm 0.50$ & $1.74 \pm 0.62$ & -16.541 & .000 \\
\hline Depression & $1.47 \pm 0.52$ & $1.61 \pm 0.65$ & -9.470 & .000 \\
\hline Anxiety & $1.44 \pm 0.49$ & $1.61 \pm 0.62$ & -12.192 & .000 \\
\hline Hostility & $1.30 \pm 0.44$ & $1.38 \pm 0.49$ & -6.529 & .136 \\
\hline Terror & $1.74 \pm 0.50$ & $1.72 \pm 0.62$ & 1.492 & .052 \\
\hline Bigotry & $1.62 \pm 0.52$ & $1.59 \pm 0.54$ & 1.941 & \\
\hline Psyc & & & & \\
\hline
\end{tabular}

\subsection{Single Factor Analysis of WI-FI Usage Affecting the Total Average Score of scl-90}

\subsubsection{Variable Assignments}

First, the possible conditions (alternative answers) of the variables that may affect the total average score of scl-90 are assigned, as shown in table 2 . 
Table 2 Variable Assignment of Factors Affecting WI-FI

\begin{tabular}{|c|c|}
\hline Item & Options and values \\
\hline 1. Major time periods of WI-FI Use & 1=morning,2=noon,3=afternoon,4=evening \\
\hline 2. Most attractive aspects of WI-FI & $1=$ convinence, $2=$ economy, $3=$ safety \\
\hline 3.Whether the phone support WI-FI & $1=$ No, $2=$ Yes \\
\hline 4.Major tools for accessing Internet with WI-FI & 1=smart phone, 2=iPad, 3=notebook, 4=desktop \\
\hline 5.Major places for accessing Internet with WI-FI & $\begin{array}{c}1=\text { classroom, } 2=\text { library, } 3=\text { dormitory, } 4=\text { other public } \\
\text { places }\end{array}$ \\
\hline 6. Whether to use WI-FI in class & $1=$ No, $2=$ Yes \\
\hline 7. Average time spending on WI-FI every day & $1=0 \sim 2 \mathrm{~h}, 2=3 \sim 4 \mathrm{~h}, 3=5 \sim 6 \mathrm{~h}, 4=$ over $7 \mathrm{~h}$ \\
\hline 8. Knowledge of WI-FI & $1=$ =little, $2=$ some, $3=$ much \\
\hline 9.Average frequency of using WI-FI & 1=once, $2=$ twice, $3=$ three times, $4=$ over three times \\
\hline 10. What do you do with WI-FI? & $\begin{aligned} \text { =play games, } 2 & =\text { chat of make friends, } 3=\text { entertainment, } \\
4 & =\text { news and information, } \\
5 & =\text { work and study, } 6=\text { others }\end{aligned}$ \\
\hline $\begin{array}{l}\text { 11. Is there a wi-fi signal transmitter installed in the } \\
\text { dormitory? }\end{array}$ & $1=$ No, $2=$ Yes \\
\hline 12.Free wi-fi software for personal computers & $1=$ No, $2=$ Yes \\
\hline 13.Are you in a hurry to get WI-FI in public? & $1=$ No, $2=$ Yes \\
\hline 14. Neck and shoulder strain & 1=No;2=yes, a bit tired;3=Yes, very serious \\
\hline 15. Lumbar involvement & 1=No; $2=$ Yes, stiff at the waist; $3=$ Yes, a bit pain \\
\hline 16. Eye strain & $1=$ as normal, $2=a$ bit uncomfortable, $3=$ sore eyes, $4=$ pain \\
\hline 17. Dependence on WI-FI & $1=$ No, not at all;2=seldom; 3=yes, very much \\
\hline 18. Use WI-FI to avoid problems or relieve emotions & 1=Never; 2=Seldom; 3=usually; 4=always \\
\hline 19. Causes of college students' WI-FI addiction & $\begin{array}{c}\text { 1=poor self-control; } 2=\text { =Family estrangement; } 3=\text { pressure } \\
\text { of study; } \quad 4=\text { The bad social atmosphere, and un-strict } \\
\text { management in Internet cafes; } 5=\text { others, please explain in } \\
\text { details }\end{array}$ \\
\hline
\end{tabular}

\subsubsection{Single Factor Analysis of WI-FI Usage Affecting the Total Average Score of scl-90} Table 3 Single Factor Analysis of WI-FI Usage Affecting the Total Average Score of scl-90

\begin{tabular}{lcc}
\multicolumn{1}{c}{ Items } & $\boldsymbol{t}$ & $\boldsymbol{P}$ value \\
\hline 1. Major time periods of WI-FI Use & 8.430 & $<.001$ \\
2. Most attractive aspects of WI-FI & 1.494 & 0.225. \\
3. Whether the phone support WI-FI & -4.641 & $<.001$ \\
4.Major tools for accessing Internet with WI-F & 3.109 & 0.029 \\
5. Major places for accessing Internet with WI-FI & 11.749 & $<.001$ \\
6. Whether to use WI-FI in class & -8.031 & $<.001$ \\
7. Average time spending on WI-FI every day & -20.009 & $<.001$ \\
8. Knowledge of WI-FI & 25.287 & $<.001$ \\
9. Average frequency of using WI-FI & -2.226 & 0.084 \\
10. What do you do with WI-FI? & 1.372 & 0.241 \\
11. Is there a wi-fi signal transmitter installed in the dormitory? & -1.115 & 0.265 \\
12. Free wi-fi software for personal computers & -4.528 & $<.001$ \\
13. Are you in a hurry to get WI-FI in public? & -1.296 & 0.195 \\
14. Neck and shoulder strain & -44.941 & $<.001$ \\
15. Lumbar involvement & -19.034 & $<.001$ \\
16. Eye strain & -40.946 & $<.001$ \\
17. Dependence on WI-FI & -51.637 & $<.001$ \\
18.Use WI-FI to avoid problems or relieve emotions & -52.308 & $<.001$ \\
19. Causes of college students' WI-FI addiction & 3.875 & 0.004 \\
\hline
\end{tabular}


Table 3 shows that, in addition to "the most attracting aspects of WI-FI", "major activities with WI-FI Internet", "whether the dormitory equipped with WI-FI signal transmitter", "whether in public use wi-fi" and so on had no significant effect of SCL - 90 total divide outside, the rest of the 15 items of SCL - 90 total share has significant effect.

\subsubsection{Multivariate Linear Regression Analysis of WI-FI Usage Conditions Affecting the Overall Mean of scl-90}

Table 4 shows, the major influencing factors of college students' mental health level (SCL - 90 total split) were the following aspects: the understanding of WI-FI, The purpose of surfing the Internet (with WI-FI access to the Internet to avoid trouble), degree of psychological dependence on WI-FI, whether to install wi-fi software, used in mobile phone support wi-fi, eyes, neck involvement degree, the degree of lumbar spine involvement, main places of using WI-FI, WIFI hours a day on average, whether using WI-FI in class time.

Table 4 Multivariate Linear Regression Analysis of WI-FI Usage Conditions Affecting the Overall Mean of scl-90

\begin{tabular}{|c|c|c|c|c|c|c|c|c|}
\hline & \multicolumn{2}{|c|}{$\begin{array}{l}\text { Unstandardized } \\
\text { Coefficients }\end{array}$} & \multicolumn{3}{|c|}{ Standard coefficient } & \multicolumn{3}{|c|}{ Relevance } \\
\hline & B & Standard error & $\begin{array}{c}\text { Trial } \\
\text { version }\end{array}$ & $\begin{array}{c}\mathrm{T} \\
\text { value }\end{array}$ & $\begin{array}{c}\mathrm{P} \\
\text { value }\end{array}$ & Zero-order & Leaning & Part \\
\hline (Constant) & 2.115 & .099 & -- & 21.290 & $<.001$ & -- & -- & - \\
\hline $\begin{array}{l}\text { Using WI-FI to avoid worries and } \\
\text { relieve emotions }\end{array}$ & .118 & .023 & .170 & 5.198 & $<.001$ & .350 & 151 & .134 \\
\hline Psychological dependence on WI-FI & .089 & .015 & .163 & 5.882 & $<.001$ & .287 & .170 & .151 \\
\hline Shoulder and neck involvement & .069 & .016 & .132 & 4.637 & $<.001$ & .315 & .117 & .103 \\
\hline Eye involvement & .064 & .016 & .126 & 4.012 & $<.001$ & .335 & .140 & .136 \\
\hline Knowledge about WI-FI & -.119 & .022 & -.142 & -5.419 & $<.001$ & -.115 & -.157 & -.139 \\
\hline Average hours on WI-FI every day & .067 & .025 & .072 & 2.696 & .007 & .138 & .079 & .069 \\
\hline Lumbar involvement & .070 & .023 & .096 & 3.026 & .003 & .294 & .089 & .078 \\
\hline Major places of using WI-FI & .072 & .024 & .079 & 2.974 & .003 & .034 & .087 & .076 \\
\hline Time periods of using WI-FI & -.070 & .022 & -.082 & -3.161 & .002 & -.137 & -.092 & -.081 \\
\hline Using WI-FI in class time or not & .041 & .013 & .082 & 3.156 & .002 & .085 & .092 & .081 \\
\hline WI-FI software & .035 & .013 & .070 & 2.643 & .008 & .036 & .070 & .061 \\
\hline Cell phone supports WI-FI or not & .066 & .028 & .063 & 2.383 & .017 & .154 & .077 & .068 \\
\hline
\end{tabular}

\section{Discussion}

The score of somatization and interpersonal sensitivity in scl-90 was significantly higher than the national norm ${ }^{[10]}$. In the five factors of OCD, depression, anxiety, hostility and terror, students in the research scored significantly lower than the national norm, prompt this group of college students' mental health status in the national college students in good level.

All the college students in this group use WI-FI every day. Among them, $80.32 \%$ of college students use WI-FI for more than 3 hours per day on average, which is significantly higher than the period when WI-FI was not popular ${ }^{[11]}$. According to Young's diagnostic criteria, they are high-frequency network users. Among them, the incidence of Internet addiction was $17.12 \%$, which was consistent with the results of Huang Hai and $\mathrm{An} \mathrm{Xi}^{[5,12]}$, higher than the research results by Bao Binggang and Lin Angui. It may be related to the sampling area. It is suggested that the use of WI-FI has become the main activity for college students in their daily life (especially after class), and its main purpose is not to study, but to play games, chat, watch movies and other recreational activities ${ }^{[6,11-12]}$.

This study showed that $28.7 \%$ of college students were in mental sub-health state $(>2$ in total score of scl-90), and problems such as somatization, coercion, interpersonal sensitivity, depression and anxiety were more common. At the same time, there were somatic problems in this group, including neck, waist and eyesight, which were consistent with previous research results ${ }^{[15-17]}$.

Multivariate regression analysis results show that college students' mental health level (SCL - 90 total split) were the major influencing factors of the following aspects: individual understanding of wi-fi and Internet purposes (for example, using WI-FI to escape trouble), the degree of 
psychological dependence on WI-FI, whether to install WI-FI software, personal computers used in the mobile phone whether to support the WI-FI, eyes, neck involvement degree of involvement degree, the degree of lumbar spine involvement, the time period of use, average daily WI-FI length, etc.

The correct knowledge of WI-FI is a protective factor for mental health. According to Davis's cognition-behavior model [18], the psychological basis of individuals' unreasonable use of the Internet is the non-adaptive cognition of the Internet. Internet technology, including WI-FI, has two sides[18]. On the one hand, it brings many benefits to individuals, such as broadening their horizon, increasing their knowledge, expanding their circle of friends and enhancing their confidence. On the other hand, the characteristics of network, especially WI-FI, such as its vitality, interactivity, real-time and convenience, are easy to make individuals become dependent and bring negative effects on their life and study. If individuals can understand the two sides of WI-FI correctly, it will be beneficial to use WI-FI scientifically and control network behaviors reasonably.

It is a common and major reason for college students to avoid the real troubles, which is consistent with previous research results ${ }^{[19]}$. This is because the Internet can make the individual reality problems and troubles stop on the Internet for individuals to contact the real problem, they will get to the Internet as the only way to solve the problem, and WI-FI availability (e.g., a complete coverage of WI-FI, have free wi-fi software installation, the use of mobile phone support WI-FI, etc.) can make the individual ${ }^{[2-3]}$ anywhere online, further contribute to its tendency to surf the Internet to escape reality problem. This practice of evading reality with WI-FI, in turn, hinders individuals from facing reality and solving problems properly, aggravating psychological distress and worsening mental health. Therefore, individuals can only extend the Internet time, gradually increase the chances of the Internet, and even in some inappropriate places such as classroom, public places and not the right time, class, or at the meeting to do morning reading (morning exercise, also can't control to get to the Internet, thus forming psychological dependence of WI-FI ${ }^{[18]}$. This study also proves this point.

Previous studies have shown that high frequency Internet users are extremely common among college students, and the sub-health problem is also serious. High frequency Internet use is closely related to the sub-health of college students ${ }^{[15-17]}$. The sub-health incidence rate of network high-frequency users is higher than that of normal network users, and the scores of depression, anxiety, sensitivity and tension of network high-frequency users are significantly higher than that of normal network users ${ }^{[20]}$. With the increase of Internet dependence, the incidence and severity of depression, anxiety and somatic symptoms increase linearly ${ }^{[21]}$. This study also confirms this. Whether there is a causal relationship between high frequency Internet use and sub-health remains to be determined in subsequent studies.

Several factors such as the degree of neck and shoulder involvement, degree of eyes involvement, the degree of lumbar spine involvement, time of WI-FI use a day on average to a certain extent, reflects the individual use of WI-FI frequency and the degree of dependence on WI-FI, prompt the more the individuals' WI-FI use frequency, the deeper the degree of dependence on WI-FI, the more vulnerable to negative effects on their mental health.

This study found that the activity of the online content (such as games, chatting or making friends, entertainment, getting news and information, work or study, etc.) influence on college students' mental health. There were no significant differences and the results do not agree with Peng Yang results [22]. The reason may be that the study of Peng Yang was carried out 9 years ago, which is different from this study. And his sample was limited to engineering students, could not represent all college students. The results of this study suggest that regardless of the activity the individual do on the net, as long as their aim is to escape from reality problem (online problems can also be used to escape from reality of learning), their mental health will be affected. 


\section{Acknowledgement}

Fund: Key Cultivation Project of Scientific Research Fund of Guangdong Medical University in 2016(No. Z2016007)

\section{References}

[1] Ma Xin, Wang Shengkai, "Ran Chenguang. Research on Problems to Be Solved in Developing WI-FI Network” [J]. China Internet. 2015, 13(2): 43-45.

[2] University of International Business and Economics. "Building a High-speed, Secure and Fully Covered Wireless Campus Network” [J]. CHINA EDUCATION NETWORK. 2012, 9(10): 49-50.

[3] Su Chengyuan, Xie Yiwen, Chen Youpeng. "Study on the Influence of the Era of WI-FI and Intelligence on College Students' Learning” [J]. Industrial \& Science Tribune. 2016, 15(17): 106-107.

[4] Nalwa K, Anand AP. Internet Addition in students: a cause of Concern [J]. Cyberpsychol Behav, 2003, 6(6): 653.

[5] Huang hai, Hou Jianxiang, Yuli. College Students' Internet and Mobile Phone Dependence and Its Correlation with Mental Health Status[J]. Chinese Journal of School Health. 2014, 35(11): 1654-1658.

[6] Yin Xiaojian, Du Jianqiang, Ji Liu. A study on the changing trend of physical health of Chinese college students [J].2012, 35(9): 79-85.

[7] Zhang Hu, Yu Ruifu. Research on the negative influence of mobile phone on college students [J]. Fujian Forum. 2008, 25(2): 113-115.

[8] Yu Chunyan, Xie Hao. Health survey and intervention study on college students with Internet addiction[J]. Zhejiang Sport Science. 2010, 32(3): 100-104.

[9] Wang Xiangdong. Handbook of Mental Health Assessment Scale (updated version)[M]. Beijing: China mental health journal press. 1999: 31-34.

[10]Zhong Wenshan, Li Lu. Research on the construction of new norm of scl-90 for college students nationwide [J]. The Chinese School Doctor. 2009, 23(3): 251-256.

[11]Liu Jihong, Sun Hongxin, Chenying. Investigation and analysis on characteristics of college students' network behavior [J]. Higher Education Exploration. 2007, 23(3): 117-121.

[12] An Xi. A study on college students' network dependence, network use motivation and family function [J]. China Journal of Health Psychology. 2012, 20(2): 296-299.

[13]Bao Ruigang, Li Yanyan, Tao Shuman. The relationship between Internet addiction video time and mental health of a medical university student.[J] Chinese Journal of School Health. 2014, 35(7): 100-1004.

[14]Lin Angui. Effects of Internet addiction on physical health of male college students [J]. Sichuan Journal of Anatomy. 2014, 22(1): 7-10.

[15]Tian Huaxi. Study on the influence of wi-fi environment on college students' physical and mental health -- a case study of Zhengzhou University [D]. Zhengzhou University, 2016.

[16]Dixit S, Shukla H, Bhagwat A, et al. A study to evaluate mobile phone dependence among students of a medical college and associated hospital of central India [J]. Indian J Community Med, 2010, 35(2): 339-341.

[17]Song Liang, Wang Lei.The influence of mobile phone dependence on the healthy lifestyle of college students[J]. Journal of Xinzhou Normal University. 2014, 30 (5): 132-134.

[18]Davis A. A cognitive - behavioral model for pathological internet use (PIU). Catalyst. [Available online at http://www. Victoriapoint. com/Y2K_anxiety. html, 1999.

[19]Bao Wulijicang, Jiang Yongzhi. The relationship between the online psychological needs of college students and the excessive use of mobile network[J]. Chinese Health Service Management. 2017, 33(3): 231-235.

[20]Zhou Lingling. The correlation between Internet addiction and sub-health in college students[J]. 
2006, 33(7): 1206-1207.

[21] Ma Jie, Li Rui, Zhu Chuanfang. The relationship between Internet addiction and mental health of adolescent students [J]. Prevention and control of chronic diseases in China. 2008, 16(2): 161-165.

[22]Peng Yang. The relationship between network behavior and mental health of college students [J].Chinese journal of health psychology.2008, 16(8): 875-880. 